[2] Tanaka S, et al. Single session of transcranial direct current stimulation transiently increases knee extensor force in patients with hemiparetic stroke. Neurorehabil Neural Repair. 2011;25:565-9.

Acknowledgements: This work was supported by the Conselho Nacional de Desenvolvimento Científico e Tecnológico (CNPq) 303.379/2018-9, Fundação de Amparo à Pesquisa do Estado de São Paulo (FAPESP) 2019/11776-6, Faculdade de Medicina da USP/SP to SKS.

Disclosure of Interests: None declared

DOI: 10.1136/annrheumdis-2021-eular.3723

\section{POS0888 EPIDEMIOLOGICAL AND CLINICAL DIFFERENCES BETWEEN ANTI-MDA5 PHENOTYPES: DATA FROM A LARGE COHORT (MEDRA5) STUDY}

E. Trallero-Araguás ${ }^{1}$, F. Romero ${ }^{2}$, I. Castellví ${ }^{3}$, V. Ortiz-Santamaria ${ }^{4}$ S. Castañeda ${ }^{5}$, O. Sanchez Pernaute ${ }^{2}, X$. Solanich ${ }^{6}$, R. Coto-Hernández ${ }^{7}$, R. Garcia de Vicuna ${ }^{5}$, Á. Robles Marhuenda ${ }^{8}$, I. Rodriguez-Pubto ${ }^{9}$, M. E. RuizLucea $^{10}$, L. Sáez-Comet ${ }^{11}$, A. Selva-O'callaghan ${ }^{12}$ on behalf of MEDRA5 (Spanish anti-MDA5 syndrome Register) group (listed contributors at the end of the abstract). ${ }^{1}$ Hospital Vall d'Hebron, Rheumatology, Barcelona, Spain; ${ }^{2}$ Hospital Universitario Fundación Jiménez Díaz, Rheumatology, Madrid, Spain; ${ }^{3}$ Hospital Santa Creu i Sant Pau, Rheumatology, Barcelona, Spain; ${ }^{4}$ Hospital General de Granollers, Rheumatology, Granollers, Spain; ${ }^{5}$ Hospital La Princesa, Rheumatology, Madrid, Spain; ${ }^{6}$ Hospital de Bellvitge, Internal Medicine, Hospitalet de Llobregat, Spain; ${ }^{7}$ Hospital Universitario Central de Asturias, Internal Medicine, Oviedo, Spain; ${ }^{8}$ Hospital Universitario La Paz, Internal Medicine, Madrid, Spain; ${ }^{9}$ Hospital Universitario Mutua de Terrassa, Internal Medicine, Terrassa, Spain; ${ }^{10}$ Hospital Universitario Basurto, Rheumatology, Bilbao, Spain; ${ }^{11}$ Hospital Miguel Servet, Internal Medicine, Zaragoza, Spain; ${ }^{12}$ Hospital Vall d'Hebron, Internal Medicine, Barcelona, Spain

Background: Idiopathic inflammatory myopathies are a heterogenous group of systemic autoimmune diseases. Several phenotypes have been linked to specific autoantibodies. Clinically amyopathic dermatomyositis with rapidly progressive interstitial lung disease, the most severe form of ILD, is associated with the antiMDA5 antibodies. However not all the patients with dermatomyositis and antiMDA5 positive antibodies develop this severe condition.

Objectives: We aim to define different phenotypes from a large cohort of patients diagnosed with dermatomyositis who were positive to anti-MDA5 antibodies. Methods: We retrospective analyzed the clinical and immunological data of 90 anti-MDA5 patients [50 female, $55.6 \%$, mean (SD) age at diagnosis 47 (15.4) yrs.] with dermatomyositis recruited from a multicenter register in Spain (MEDRA5) including 30 hospitals. All the patients fulfill de International Myositis Classification Criteria (EULAR/ACR) for dermatomyositis (score $>90 \%$ ). AntiMDA5 were detected by means of commercial immunoblot (EUROIMMUN $\left.{ }^{\odot}\right)$. The chi-square test was used to assess the relationships between qualitative variables. The Kruskal-Wallis test was used to compared medians between groups. Results: Sixty-six patients $(73.3 \%)$ were diagnosed with clinically amyopathic dermatomyositis. Three different phenotypes linked with the anti-MDA5 antibody were identified. Group 1: patients with rapidly-ILD phenotype (28 patients, $31.1 \%$ ), group 2: antisynthetase-like phenotype (23 patients, $25.5 \%$ ), and group 3: non-ILD phenotype (39 patients, 43.3\%). Clinical and immunological comparison between the groups disclosed that age at disease onset was higher (median, IQR) in patients from group 1 [53 (43-60)] vs. group 2 [46 (40-56)] or group 3 [42(41-51)] $(p=0.01)$; disease onset was more frequent in spring in patients from group $1(46.5 \%)$ than in the rest of the groups $(21.7 \%$ and $28.9 \%)$ $(p<0.01)$. Cancer was detected in 7 patients, only associated with myositis in 3 cases ( 3 years interval between cancer and dermatomyositis) without significant differences between phenotypes. Vasculitis (one case ANCA positive) was detected in 9 cases ( 6 limited to skin, 1 renal and 1 intestinal), 6 of them in the group 3 (statistical significance, in comparison with group 1 and 2, $p<0.01$ ). Mortality rate was higher in group $1(51.9 \%, 16$ out of 17 due to refractory respiratory failure) vs group $2(12.5 \%)$ or $3(0 \%)(p<0.001)$. Anti Ro52 positivity was more frequent in group $1(65.4 \%)$ vs. group $2(25 \%)$ or $3(35.5 \%)(p<0.017)$, although it did not reach statistical significance in terms of mortality $(p=0.173)$ or patients admitted in the intensive care unit $(p=0.173)$. Mechanic hands were more frequent in group $2(40.6 \%)$ than in groups $1(25 \%)$ and $3(34.4 \%)(p=0.05)$. Fever was significantly most frequent in group $1(52.6 \%)$ than in group $2(21.1 \%)$ and $3(26.3 \%)(p=0.001)$. Other clinical or immunological features such as arthritis, myositis, or the number of characteristic skin lesions among others were not more frequent in one group or another.

Conclusion: Three different phenotypes of patients positive to anti-MDA5 were identified. The presence or not of ILD, or the different type (rapidly progressive or not) of ILD were the main feature that allow to differentiate these phenotypes, which are relevant in clinical practice.

\section{REFERENCES:}

[1] Allenbach Y, Uzunhan Y, Toquet S, et al; French Myositis Network. Different phenotypes in dermatomyositis associated with anti-MDA5 antibody: Study of 121 cases. Neurology. 2020;95: e70-e78.

Acknowledgements: List of contributors of MEDRA5 group: Aguilar-García J (Internal Medicine, Hospital Costa del Sol, Marbella), Carrión-Barberá I (Rheumatology, Hospital del Mar, Barcelona), Cobo-Ibañez T (Rheumatology, Hospital Infanta Sofía, San Sebastián de los Reyes), de Escalante-Yangüela B (Internal Medicine, Hospital Clínico Lozano Blesa, Zaragoza), Fonseca-Aizpuru EM (Internal Medicine, Hospital de Cabueñes, Gijón), González-Cubillo L (Intensive Medicine, Hospital Universitario de Cruces, Barakaldo), González-Gay MA (Rheumatology, Hospital Marqués de Valdecilla, Santander), Prieto-González S (Internal Medicine, Hospital Clinic, Barcelona), Ruiz-Román A (Rheumatology, Hospital Universitario Virgen del Rocío, Sevilla), Calero-Paniagua I (Internal Medicine, Hospital Virgen de la Luz, Cuenca), Callejas-Rubio JL (Internal Medicine, Hospital Clínico San Cecilio, Granada), Gil-Vila A (Internal Medicine, Hospital Vall d'Hebron, Barcelona), de Miguel-Campo B (Internal Medicine, Hospital Doce de Octubre, Madrid), García-Sevilla R (Pneumology, Hospita General Universitario de Alicante, Alicante), Iriarte-Fuster A (Internal Medicine, Hospital de Bellvitge, Hospitalet de Llobregat), Jovani-Casano V (Rheumatology, Hospital General Universitario de Alicante, Alicante), Lozano-Rivas N (Rheumatology, Hospital Virgen de la Arritxaca, Murcia), Martín-Gascón M (Internal Medicine, Hospital Morales Meseguer, Murcia), Martinez-González O (Rheumatology, Hospital Universitario de Salamanca, Salamanca), Monteagudo-Jiménez M (Internal Medicine, Hospital Parc Taulí, Sabadell), Mora-Ortega GM (Pneumology, Hospital Universitario Infanta Sofía, San Sebastián de los Reyes), Moral-Moral Pedro (Internal Medicine, Hospital Universitari i Politecnic La Fe, Valencia), Pérez-De Pedro I (Interna Medicine, Hospital Regional Universitario de Málaga, Málaga), Picazo-Talavera MR (Rheumatology, Hospita del Sureste, Madrid), Rubio-Rivas M (Internal Medicine, Hospital de Bellvitge, Hospitalet de Llobregat)

Disclosure of Interests: None declared

DOI: 10.1136/annrheumdis-2021-eular.3837

\section{\begin{tabular}{|l|l|l|}
\hline POS0889 CO PRESCRIPTION OF ANTI-ACID THERAPY \\
\hline
\end{tabular} REDUCES THE BIO AVAILABILITY OF MYCOPHENOLATE MOFETIL IN SYSTEMIC SCLEROSIS PATIENTS}

G. Alex ${ }^{1}$, S. K C ${ }^{2}$, D. Reachel Varghese ${ }^{1}$, S. Babu A S ${ }^{2}$, R. Reji ${ }^{2}$, P. Shenoy ${ }^{2}$.

${ }^{1}$ Center for Arthritis and Rheumatism Excellence (CARE), Rheumatology, Kochi, India; ${ }^{2}$ Center for Arthritis and Rheumatism Excellence (CARE), Rheumatology, Kochi, India

Background: Mycophenolate mofetil (MMF) is an effective treatment option for systemic sclerosis (SSC). However, many patients require co administration of proton pump inhibitors (PPI) or $\mathrm{H} 2$ receptor blockers (HRB) because of significant gastrointestinal manifestations in SSC. Co-treatment with $\mathrm{PPI}$ or HRB have shown to be associated with reduced drug exposure in post-transplant patients. ${ }^{1,2}$ There is scarcity of data among patients with SSC. We evaluated the drug concentration of MMF over 12 hours of exposure and assessed the impact of ranitidine and PPI in twenty patients with SSC

Objectives: To assess the effect of esomeprazole or ranitidine on the bioavailability of MMF in SSC patients who are on a stable dose of MMF.

Methods: Twenty SSC patients, who were on a stable dose of MMF $(1.5-3 \mathrm{~g})$ for the past 3 months were selected for the study after obtaining informed written consent. All patients were given either MMF (without PPI or HRB), MMF + esomeprazole, MMF + ranitidine for one month each. At the end of each month, EDTA plasma samples were collected at various time points including $0,1 / 2,1,1 \frac{1}{2}, 2,2 \frac{1}{2}, 3,4,5,6,8$ and 12 hours following drug administration to determine the 12-hour area under curve (AUC) of mycophenolic acid (MPA) levels. Estimation of MPA levels was carried out using reverse phase high performance liquid chromatography (HPLC). Total gas trointestinal score was calculated at the end of each month using UCLA Scleroderma Clinical Trial Consortium GIT 2.0 scoring. To compare the mean AUC, linear mixed effect model was fit by considering treatment as the fixed effect and subject as the random effect. MMF was set as the reference treatment for the other three treatments and these were analysed together using Linear mixed effect model.

Results: All patients were females with mean age of 45 years. Addition of either ranitidine or esomeprazole significantly reduced the mean AUC and $\mathrm{C}$ max of the MMF over 12-hour time period. On the other hand, PPI or HRB helped in reduction of the total GI score at the end of 1 month. Details of pharmacokinetics are depicted in the table 1. 